\title{
Percepción del impacto de cerdos cimarrones (jabalíes) sobre la producción agropecuaria de Argentina
}

\author{
Marcos, A. ; Carpinetti, B. 2.; Ferro N.'; Aronowicz T.'; Dassa L. ${ }^{1}$ \\ ${ }^{1}$ Servicio Nacional de Sanidad y Calidad Agroalimentaria, Colón 367, Buenos Aires. \\ ${ }^{2}$ Instituto de Ciencias Sociales y Adm., Univ.A.Jauretche, F.Varela, Buenos Aires. \\ E-mail: amarcos@senasa.gob.ar
}

\begin{abstract}
Resumen
Marcos, A.; Carpinetti, B.; Ferro, N.; Aronowicz, T.; Dassa, L.: Percepción del impacto de cerdos cimarrones (jabalies) sobre la producción agropecuaria de Argentina. Rev. Vet. 31: 2, 131-136, 2020. Para conocer la percepción de los productores agropecuarios respecto al impacto del jabalí sobre la actividad productiva y el ambiente, se elaboró una encuesta destinada a tal fin en Argentina, realizándose un análisis descriptivo y estadístico de los resultados. Los productores que respondieron la encuesta se dedicaban en su mayoría a la actividad ganadera ( $96 \%$ ) y el $69 \%$ realizaba actividad mixta (ganadera y agrícola). El $39 \%$ de los productores detectó daños a cultivos provocados por jabalíes, principalmente en cereales (maíz, sorgo, trigo, avena, cebada, centeno) y en oleaginosas (soja y girasol), sin que se hayan demostrado diferencias significativas entre el daño y el tipo de cultivo $(p=0,88)$. Otros daños fueron deterioros a la infraestructura (30\%), animales atacados $(20 \%)$ y consumo del alimento del ganado (45\%). El 37\% conoció personas involucradas en accidentes automovilísticos relacionados con jabalíes. De una escala de 1 (dañino) a 10 (beneficioso), el puntaje promedio del jabalí fue de 3,16 (desvío estándar $=2,27$ ). En algunas zonas este animal fue considerado beneficioso debido a su uso como especie cinegética (cacería). No obstante, el jabalí resulta ser un problema relevante para gran parte de los productores agropecuarios pues se lo asocia a pérdidas económicas importantes. Considerando que los productores son destinatarios directos del impacto benéfico que tendría la disminución de la cantidad de jabalíes, su concientización respecto al tema resultaría alentadora, favoreciéndose el desarrollo de controles poblacionales de la especie, involucrándose a las autoridades ambientales y las vinculadas a la industria agropecuaria.
\end{abstract}

Palabras clave: jabalí, percepción, impacto, productores agropecuarios, Argentina.

\begin{abstract}
Marcos, A.; Carpinetti, B.; Ferro, N.; Aronowicz, T.; Dassa, L.: Perception of the impact of wild boar (feral pigs) on agriculture and livestock production in Argentina. Rev. Vet. 31: 2, 131-136, 2020. To evaluate the perception of agricultural and livestock producers regarding the impact of wild boar on their activity and the environment, a survey was conducted in Argentina. For better representativity, the survey was distributed in different ways. A descriptive and statistical analysis of the results was performed. The producers who answered the survey are mostly engaged in livestock activity (96\%) but $69 \%$ had both livestock and crops. $39 \%$ of the producers detected damage to those crops, mainly in cereals (corn, sorghum, wheat, oats, barley and rye) and in oilseeds (soy and sunflower), without significant differences associated to the type of crop $(\mathrm{p}=0.88)$. Other damages referred were infrastructure damage $(30 \%)$, animals attacked (20\%) and livestock feed consumption $(45 \%) .37 \%$ had or know someone involved in car accidents related to wild boar. From a scale of one (harmful) to ten (beneficial) the average wild boar score assigned was 3.16 (standard deviation $=2.27$ ). However, in some areas of our country they consider the species to be beneficial (significant spatial clustering) due to its use as a hunting species. The wild boar turns out to be a relevant problem for a large part of agricultural and livestock producers and it is associated with several economic losses. Considering that the producers would be benefited with the decrease in the number of wild boars their awareness is encouraging, thinking of developing population control plan. This must be a priority to work inter-institutionally in Argentina, involving environmental authorities and those linked to the agricultural and livestock industry.
\end{abstract}

Key words: wild boar, perception, impact, agricultural and livestock producers, Argentina. 


\section{INTRODUCCIÓN}

La introducción de especies más allá de su rango geográfico de distribución, ha sido caracterizada como una de las más importantes amenazas para los ecosistemas y los sistemas productivos ${ }^{18}$. Una de las especies invasoras potencialmente perjudiciales debido a su impacto en los sistemas naturales y la producción agropecuaria es el jabalí (Sus scrofa) y sus diferentes morfotipos de cerdos asilvestrados ${ }^{22}$.

El jabalí es un mamífero ampliamente distribuido en el mundo. Su rango geográfico se extiende desde el oeste europeo hasta la taiga rusa, distribuyéndose hacia el sur desde el norte de África, las costas del Mediterráneo y el Oriente Medio, a lo largo del subcontinente hindú y el sudeste asiático, hasta las islas de Sri Lanka, Taiwán, Japón y el archipiélago Sunda ${ }^{25}$.

En cuanto a las formas domésticas y ferales o cimarronas, desde los estadios tempranos de la expansión y dispersión de la civilización europea, los jabalíes extendieron ampliamente su distribución y establecieron poblaciones naturalizadas en Australasia, las Américas y Oceanía, además de en un gran número de islas oceánicas ${ }^{13,26}$.

Los impactos negativos de esta especie están asociados a las alteraciones que provocan en los suelos, la vegetación y la fauna, incluyendo las consecuencias económicas asociadas al daño a los cultivos y cría de animales, así como la transmisión de enfermedades, incluidas las zoonóticas ${ }^{5}$. Debido a ello se ha requerido la implementación de planes de control o erradicación en diferentes lugares del planeta ${ }^{4,10,14,30}$.

En Argentina han existido al menos dos olas de invasión de esta especie, dando como resultado un número importante de poblaciones de Sus scrofa, con una gran heterogeneidad genética y morfológica, que se han naturalizado y se encuentran ampliamente distribuidas en Argentina $^{3,28}$. Mientras que las poblaciones silvestres más antiguas son descendientes de cerdos domésticos liberados durante la colonización española ${ }^{3,13,24}$, otras se originaron de ejemplares del jabalí euroasiático puro, deliberadamente introducido durante la primera década del siglo XX con propósitos cinegéticos ${ }^{16,24,28}$.

El jabalí o cerdo silvestre (cimarrón) ha sido senalado como una de las especies de mayor relevancia sanitaria para la salud del hombre y de los animales domésticos. Ello se debe a su amplia distribución geográfica y su constante crecimiento poblacional, lo que favorece el contacto con el ganado doméstico y las personas ${ }^{7}$. Entre las enfermedades informadas en cerdos silvestres (cimarrones) en Argentina se encuentran brucelosis $^{7}$, leptospirosis ${ }^{8,27}$, enfermedad de Aujeszky ${ }^{1,7}$, tuberculosis ${ }^{2}$, triquinosis ${ }^{11,33-35}, \mathrm{y}$ otras parasitosis que afectan al hombre y al ganado ${ }^{9}$.

En Argentina, el impacto de la especie sobre los ambientes naturales y la biodiversidad ha sido ampliamente documentado ${ }^{15}$. Tal como se ha señalado, su carácter de "ingeniero de ecosistemas" hace que la especie genere múltiples impactos en los espacios colonizados, modificando las condiciones del suelo y de la vegetación, trasmitiendo decenas de enfermedades a la fauna autóctona y transformándose en un depredador o presa dominante que produce la modificación de las relaciones interespecíficas originales entre especies nativas ${ }^{29}$.

El Ministerio de Ambiente y Desarrollo Sustentable de Argentina realizó una evaluación económica preliminar del daño asociado a este animal, estimando costos actuales discriminando daños y reducciones productivas, resultando la pérdida anual asociada a esta especie entre U\$S 907.023.190 y U\$S 1.380.488.228 ${ }^{23}$.

Uno de los pasos iniciales para considerar la aplicación de estrategias de control poblacional de especies dañinas es la evaluación de la opinión de los posibles afectados, para identificar aliados u opositores. Una de las principales fallas en los programas de control de jabalíes se debe a que se consideran únicamente los aspectos biológicos del problema, sin contemplar los aspectos sociales ${ }^{6}$.

Los conflictos a menudo no resultan de los daños reales que provoca esta especie, sino de la percepción de los productores agropecuarios respecto a los efectos del jabalí y la apreciación de una falta de controles para solucionar el problema ${ }^{17}$. Existen en este sentido antecedentes de evaluación realizados en otros países respecto de la percepción que tienen los productores sobre el impacto del jabalí a través de encuestas ${ }^{19,31}$.

A fin de conocer la percepción de los productores agropecuarios respecto al impacto del jabalí y los cerdos asilvestrados sobre la actividad productiva y el ambiente en la República Argentina, se elaboró una encuesta destinada a tal fin. Los resultados fueron presentados a organismos oficiales y otros actores involucrados en el control poblacional de esta especie.

\section{MATERIAL Y MÉTODOS}

Se elaboró una encuesta que abarcó 29 preguntas de "respuesta cerrada" sobre el tipo de producción agropecuaria realizada por el encuestado, la presencia de jabalíes en la zona, los diferentes daños asociados a tales animales y la clasificación de la especie como dañina o beneficiosa, a criterio del informante.

La encuesta se distribuyó por diferentes vías: veterinarios locales, Ministerio de Agricultura, Ganadería y Pesca de la Nación (por esta vía específicamente a productores caprinos y ovinos), Comisión Nacional de Lucha contra las Enfermedades Porcinas (a productores porcinos) y en la página oficial de SENASA.

La información de la encuesta se obtuvo a través de los datos de productores (anónimos) y veterinarios locales. Estos últimos incluyeron la identificación del predio a fines de poder ubicar geográficamente el origen de la información (partido, departamento y provincia). Desde marzo de 2018 hasta marzo de 2019 se recopilaron 518 encuestas.

El análisis fue principalmente descriptivo, aunque algunas variables fueron comparadas a través de chi cuadrado $\left(\mathrm{X}^{2}\right)$ para detectar diferencias estadística- 
mente significativas entre grupos. Los análisis estadísticos se realizaron en la plataforma de Working in Epidemiology (winepi.net). La distribución espacial de las opiniones de los encuestados se evaluó utilizando la técnica de rastreo espacial a través del SatScan ${ }^{20}$.

El análisis espacial se llevó a cabo a fin de identificar agrupamientos de casos, es decir, áreas geográficas con mayor frecuencia de casos que la esperada si la distribución fuese aleatoria. Para identificar agrupamientos de opiniones positivas o negativas respecto al jabalí, se utilizó el modelo de Bernoulli ${ }^{21}$. La información respecto a la distribución del jabalí y la estimación de su primera aparición se representaron en mapas realizados con el programa QGIS versión 3.6.3 ${ }^{32}$.

\section{RESULTADOS}

El $74 \%$ de las encuestas pertenecían a las provincias de Buenos Aires, La Pampa, Río Negro, Corrientes, Córdoba y San Luis. El resto correspondían a Neuquén, Santa Fe, Chubut, Entre Ríos, Santa Cruz, Misiones, La Rioja, Formosa, Chaco, Catamarca, Tucumán, San Juan y Mendoza (Figura 1).

Tabla 1. Tipo de producción de los animales encuestados.

\begin{tabular}{|c|c|c|c|c|c|}
\hline especie & cantidad & $\%$ & tipo de producción & cantidad & $\%$ \\
\hline \multirow{7}{*}{ bovinos } & \multirow{7}{*}{715} & \multirow{7}{*}{68} & bovinos cría & 340 & 32.4 \\
\hline & & & bovinos invernada & 158 & 15.0 \\
\hline & & & bovinos recría & 131 & 12.5 \\
\hline & & & bovinos engorde a corral & 48 & 4.6 \\
\hline & & & bovinos cabaña & 20 & 1.9 \\
\hline & & & bovinos tambo & 12 & 1.1 \\
\hline & & & bovinos subsistencia & 6 & 0.6 \\
\hline \multirow{5}{*}{ ovinos } & \multirow{5}{*}{148} & \multirow{5}{*}{14} & ovinos cría & 112 & 10.7 \\
\hline & & & ovinos subsistencia & 20 & 1.9 \\
\hline & & & ovinos invernada & 11 & 1.0 \\
\hline & & & ovinos cabaña & 4 & 0.4 \\
\hline & & & ovinos tambo & 1 & 0.1 \\
\hline \multirow{4}{*}{ porcinos } & \multirow{4}{*}{52} & \multirow{4}{*}{5} & porcinos prod. familiar & 22 & 2.1 \\
\hline & & & porc.criadero comercial & 20 & 1.9 \\
\hline & & & porcinos subsistencia & 5 & 0.5 \\
\hline & & & porcinos invernador & 5 & 0.5 \\
\hline \multirow{5}{*}{ caprinos } & \multirow{5}{*}{47} & \multirow{5}{*}{4} & caprinos cría & 29 & 2.8 \\
\hline & & & caprinos subsistencia & 10 & 1.0 \\
\hline & & & caprinos invernada & 6 & 0.6 \\
\hline & & & caprinos cabaña & 1 & 0.1 \\
\hline & & & caprinos tambo & 1 & 0.1 \\
\hline \multirow{4}{*}{ aves } & \multirow{4}{*}{44} & \multirow{4}{*}{4} & aves de traspatio & 19 & 1.8 \\
\hline & & & aves huevo & 17 & 1.6 \\
\hline & & & aves carne & 6 & 0.6 \\
\hline & & & aves padres & 2 & 0.2 \\
\hline \multirow{2}{*}{ equinos } & \multirow{2}{*}{20} & \multirow{2}{*}{2} & equinos otras actividades & 15 & 1.4 \\
\hline & & & equinos haras o cabaña & 5 & 0.5 \\
\hline agrícultura & 13 & 1 & solo actividad agrícola & 13 & 1.2 \\
\hline apiarios & 12 & 1 & apiarios & 12 & 1.1 \\
\hline total & 1051 & & total & 1051 & - \\
\hline
\end{tabular}

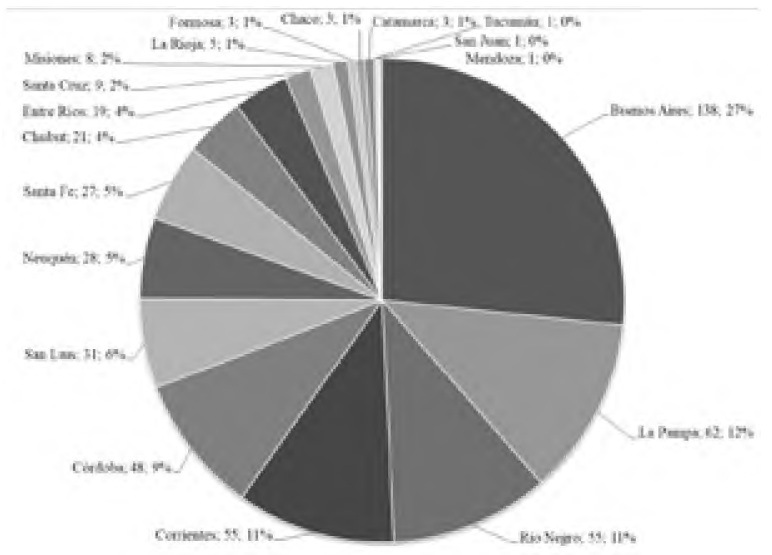

Figura 1. Distribución de las encuestas por provincias.

Los productores que respondieron la encuesta se dedicaban en su mayoría a la actividad ganadera (96\%). Sólo 13 de ellos se ocupaban exclusivamente en tareas agrícolas y 5 no respondieron la pesquisa (Tabla 1).

El $72 \%$ de los encuestados tenían al menos un cultivo en su campo (Tabla 2) y el 69\% realizaban cultivos y criaban ganado. El $68 \%$ de los encuestados se dedicaban a la ganadería bovina. Así, un productor podía realizar varias actividades productivas.

Tabla 2. Cultivos plantados por los encuestados.

\begin{tabular}{|c|c|c|}
\hline tipo de cultivo & cantidad & $\%$ \\
\hline maíz & 239 & 23,43 \\
\hline sorgo & 154 & 15,10 \\
\hline soja & 136 & 13,33 \\
\hline trigo & 114 & 11,18 \\
\hline avena & 113 & 11,08 \\
\hline girasol & 57 & 5,59 \\
\hline cebada & 52 & 5,10 \\
\hline centeno & 28 & 2,75 \\
\hline otros & 21 & 2,06 \\
\hline hortalizas & 19 & 1,86 \\
\hline frutas & 18 & 1,76 \\
\hline pastura natural & 12 & 1,18 \\
\hline eucaliptos & 10 & 0,98 \\
\hline ninguno & 10 & 0,98 \\
\hline verduras & 10 & 0,98 \\
\hline arroz & 9 & 0,88 \\
\hline caña de azúcar & 3 & 0,29 \\
\hline ray grass y setaria & 3 & 0,29 \\
\hline alfalfa & 2 & 0,20 \\
\hline pinar & 2 & 0,20 \\
\hline yerba mate & 2 & 0,20 \\
\hline garbanzos & 1 & 0,10 \\
\hline intersiembra mallines & 1 & 0,10 \\
\hline olivos & 1 & 0,10 \\
\hline pecan & 1 & 0,10 \\
\hline trébol & 1 & 0,10 \\
\hline vid & 1 & 0,10 \\
\hline TOTAL & 1020 & \\
\hline
\end{tabular}




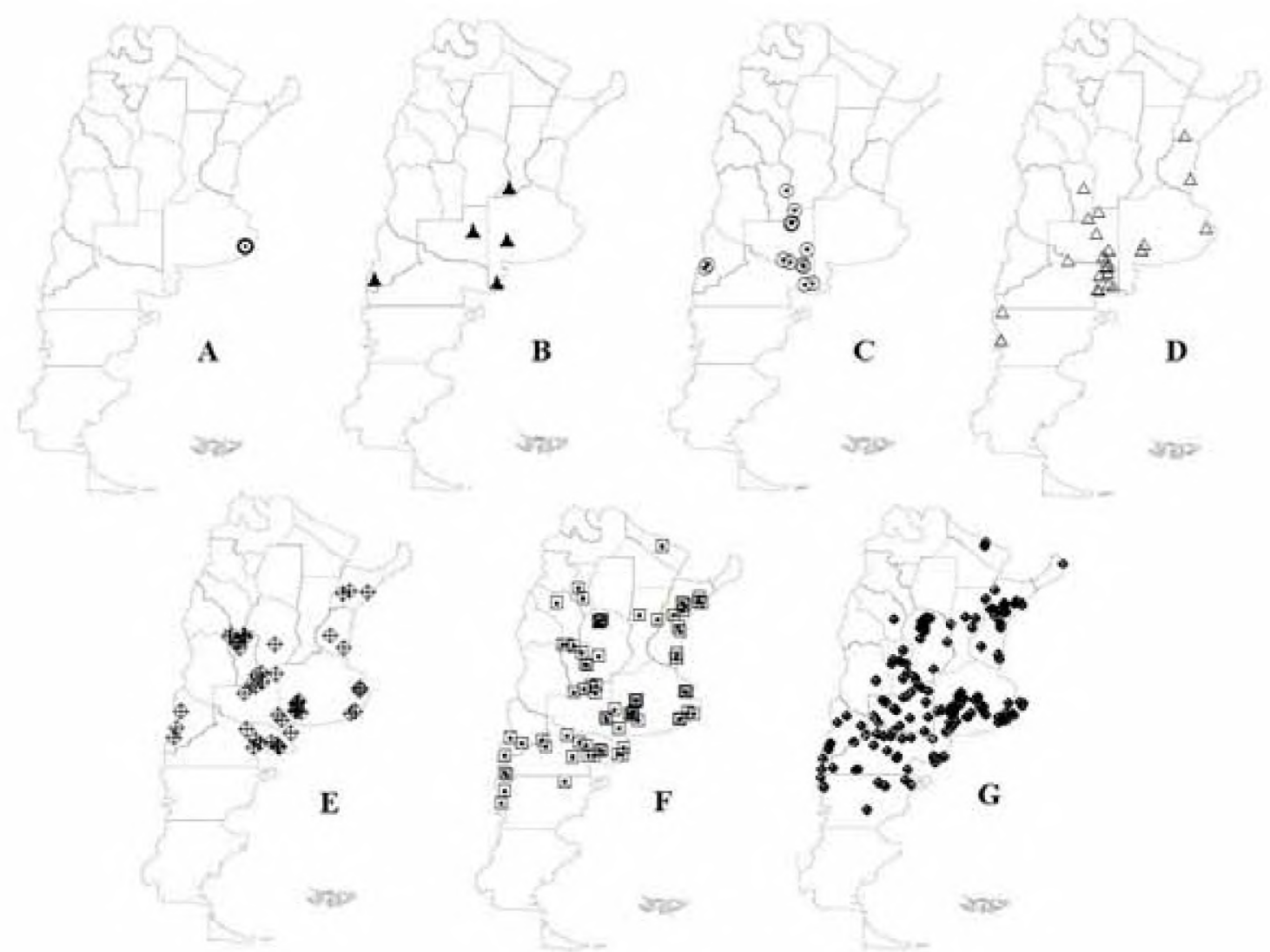

Figura 2. Primera observación de jabalíes. A: 1951. B: 1960-1969. C: 1970-1979. D: 1980-1989. E: 1990-1999. F: 2000-2009. G: 2010-2019.

El $75 \%$ de los encuestados detectó la presencia de jabalíes en su establecimiento, el $28 \%$ en grupos de más de 10 animales, y el 51\% refirió verlos frecuentemente (todos los meses). Respecto a cuándo detectaron por primera vez la presencia de jabalíes la Figura 2 permite visualizar la expansión de la especie, la cual se produjo mayoritariamente en la última década.

Respecto al impacto, el $39 \%$ de los productores detectó daños a cultivos, principalmente en cereales (maíz, sorgo, trigo, avena, cebada y centeno) y en oleaginosas (soja y girasol). Sin embargo no hubo diferencias significativas entre el daño y el tipo de cultivo ( $p$ $=0,88$ ).

Acerca de otros daños, el $30 \%$ refirió deterioros a infraestructuras provocados por jabalíes, el $20 \%$ señaló animales atacados y el $45 \%$ verificó que ellos consumían el alimento del ganado. El $37 \%$ de los productores tuvo o conoció personas involucradas en accidentes de auto relacionados con jabalíes. Por ello, el $61 \%$ de las personas encuestadas consideraron a este animal como un tema relevante.

Existe cierto desconocimiento asociado al riesgo sanitario que implica esta especie invasora. El $71 \%$ de los encuestados es conciente que ellos transmiten enfermedades zoonóticas al ser humano, pero solo el 39\% sabe que transfieren enfermedades a otros animales, siendo que existe evidencia que la enfermedad de $\mathrm{Au}-$ jeszky se encuentra presente en jabalíes de Argentina con prevalencias superiores al $60 \%{ }^{1,7}$.
Es importante que los productores relacionen al jabalí con daños a los ambientes naturales conservados y a otros animales silvestres ( $26 \%$ y $14 \%$ respectivamente). Las especies más mencionadas como afectadas son carpinchos, ciervos, tortugas, yacarés y varias especies de aves (patos, martinetas y ñandúes).

Finalmente, se pidió a los encuestados que evaluaran en una escala de 1 al 10 cuan dañino consideraban al jabalí, siendo 1 el valor que lo asumía como especie

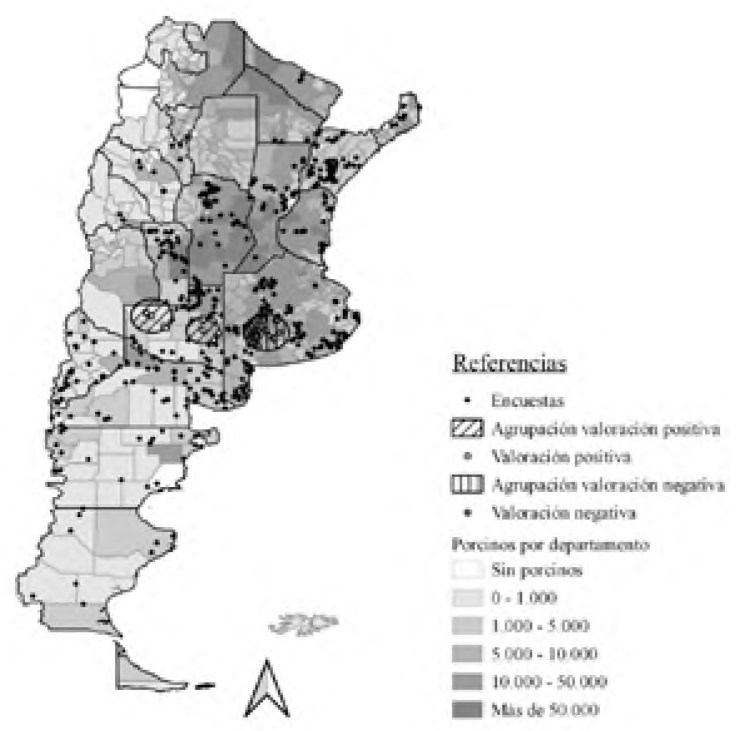

Figura 3. Agrupación espacial de valoración del jabalí y distribución de porcinos. 
dañina y 10 la opinión que lo creía beneficioso. Los resultados colocaron al animal en un puntaje de 3,16 (desvío estándar $=2,27$ ), siendo el modo 1 y la mediana 3. Los datos de percepción de la especie se analizaron espacialmente, buscando agrupaciones espaciales (clusters).

Para el caso de las valoraciones positivas (puntaje mayor o igual a 6) se detectaron dos clusters que resultaron significativos $\left(p_{1}=0,018\right.$ y $\left.p_{2}=0,05\right)$, ubicados en la Provincia de La Pampa. El primer cluster agrupó cinco encuestas en un radio de $91 \mathrm{~km}$ y el segundo ocho encuestas en un radio de $71 \mathrm{~km}$.

Para el caso de las valoraciones negativas (puntaje menor o igual a 5) se detectó un único cluster significativo $(p=0,023)$ que agrupó 37 encuestas en un radio de $98 \mathrm{~km}$. Las agrupaciones se muestran en la Figura 3 junto con la densidad de porcinos de producción de la República Argentina.

\section{DISCUSIÓN}

Las encuestas fueron obtenidas de las principales zonas productivas de la República Argentina y se encuentran representadas las actividades ganaderas y agrícolas más importantes.

Los jabalíes han incrementado sistemáticamente su rango de distribución en las últimas décadas, probablemente por la reducción de cacerías, por los cambios demográficos en la población rural, y también por la casi total ausencia de depredadores naturales en las áreas colonizadas por la especie, así como por la creciente disponibilidad de recursos forrajeros a consecuencia del incremento constante de las áreas cultivadas.

De la encuesta se desprende que existen algunas zonas en las que la especie es relativamente nueva y otras donde ya se encuentra establecida, siendo vista de manera regular y en grupos de varios animales.

Se verifica que los impactos negativos descriptos en la bibliografía ${ }^{5}$, tales como perjuicio a los cultivos, deterioro a la infraestructura de los campos, daño a los animales de producción y consumo de alimento para el ganado, se observan en nuestro país. Específicamente en lo que respecta a los cultivos, el más afectado en Argentina sería el maíz (24\%), seguido por el sorgo (15\%) y la soja (13\%).

Estos daños son diferentes en otras latitudes como Louisiana (USA), aunque el maíz y la soja continúan siendo los cultivos más afectados por los jabalíes. Así, los daños reportados al ganado resultan menores a lo detectado en nuestra encuesta ${ }^{31}$

Los resultados obtenidos en Argentina indican que los productores consideran al jabalí como un problema y son conscientes de sus impactos negativos, lo cual alienta a iniciar acciones de control poblacional de la especie. Esta situación es diferente a lo observado en Saskatchewan (Canadá), donde los productores no valoran negativamente al jabalí ni su impacto ${ }^{6}$.

En algunas zonas de nuestro país (La Pampa) consideran beneficioso al jabalí por valorar tal especie como recurso económico vinculado a empresas familiares que practican la caza deportiva como estrategia de diversificación productiva. En la mayoría de los casos, esta práctica es una actividad secundaria, complementaria a la cría de ganado vacuno ${ }^{12}$. Datos similares se registran en Australia, donde el impacto de la especie es visto como positivo entre los cazadores y debe ser considerado cuando se planifique una estrategia de control poblacional ${ }^{19}$.

No obstante, el jabalí resulta ser un problema relevante para gran parte de los productores agropecuarios de nuestro país y se lo asocia a pérdidas económicas diversas. Considerando que los productores son destinatarios directos del impacto que tendría la disminución de la cantidad de jabalíes, deben ser convocados para intervenir en los planes de control poblacional de la especie.

El desarrollo de este tema debe ser una prioridad interinstitucional en Argentina, involucrando a las autoridades ambientales y las vinculadas a la industria agropecuaria.

Agradecimientos. Se agradece a los veterinarios de SENASA, del Ministerio de Agricultura, Ganadería y Pesca de la Nación y de la Comisión Nacional de Lucha contra las Enfermedades de los Porcinos, por colaborar en la distribución y compilación de las encuestas. A Emiliano Grave y Mirta Medvescic por la colaboración en el armado de los mapas.

\section{REFERENCIAS}

1. Abate $\mathbf{S}$ et al. 2016. Enfermedad de Aujeszky en Patagonia Noreste: reporte de casos positivos en jabalí (Sus scrofa). Rev Coleg Med Vet Patagonia 29: 16-18.

2. Abate $\mathbf{S}$ et al. 2017. El jabalí como reservorio silvestre de tuberculosis en el noreste de la Patagonia argentina. $X$ Jorn Intern de Vet Práct, Mar del Plata, Argentina.

3. Acosta, DB et al. 2019. Genetic diversity and phylogenetic relationships in feral pig populations from Argentina. Mamm Biol 99: 27-36.

4. Anderson SJ, Stone CP. 1993. Snaring to control feral pigs Sus scrofa in a remote Hawaiian rain forest. Biol Conserv 63: 195-201.

5. Barrios MN, Ballari SA. 2012. Impact of wild boar (Sus scrofa) in its introduced and native range: a review. Biol Invas 14: 2283-2300.

6. Brook RK, Beest FM. 2014. Feral wild boar distribution and perceptions of risk on the central Canadian prairies. Wildlife Soc Bull 38: 486-494.

7. Carpinet TI et al. 2017. Determinación de anticuerpos contra patógenos virales y bacterianos seleccionados en la población de cerdos silvestres (Sus scrofa) de la Reserva Natural Bahía Samborombón, Argentina. Analecta Vet 37 : 21-27.

8. Cifuentes S et al. 2016. Leptospirosis en jabalí (Sus scrofa) en una zona de la Patagonia noreste. XXIX Jorn Argent Mastozoología, San Juan, Argentina. 
9. Ciocco RB et al. 2019. Endoparásitos de una población de cerdos silvestres (Sus scrofa) en Bahía Samborombón, Buenos Aires, Argentina. Rev Mex Biodiv 90: 1.

10. Clout MN, Veitch CR. 2002. Turning the tide of biological invasion: the potential for eradicating invasive species. IUCN SSC Invasive Species, Cambridge, UK 1-3.

11. Cohen $\mathbf{M}$ et al. 2010. Trichinella infection in wild boars (Sus scrofa) from a protected area of Argentina and its relationship with the presence of humans. Vet Parasit 169: 362-366.

12. Comerci ME. 2016. Avance de los cotos de caza en La Pampa ¿nuevas dinámicas territoriales? Rev Investig Cs Soc, Facult Humanid \& Cs Soc, UNAM, Argentina

13. Crosby AW. 1986. Ecological imperialism: the biological expansion of Europe, Cambridge University Press, New York, 900: 1900

14. Cruz F, Donlan CJ, Campbell K, Carrion V. 2005. Conservation action in the Galapagos: feral pig (Sus scrofa) eradication from Santiago Island. Biol Conserv 121: 473478

15. Cuevas MF, Ojeda RA, Jaksic FM. 2016. Estrategias ecológicas e impacto del jabalí en provincias fitogeográficas de Argentina con énfasis en las tierras áridas. Mastozool Neotrop 23: 239-255.

16. Daciuk J. 1978. Estado actual de las especies de mamíferos introducidos en la Subregión A raucana (Argentina) y grado de coacción ejercido en algunos ecosistemas surcordilleranos. An Parq Nac 14: 105-130.

17. Decker DJ et al. 1996. From clients to stakeholders: a philosophical shift for fish and wildlife. Management 70 : 82.

18. Hobbs RJ. 2000. Invasive species in a changing world. Island Press, $255 \mathrm{p}$.

19. Koichi K. 2012. The perceived environmental and socioeconomic impacts of feral pigs (Sus scrofa): A re-examination of their perceived pest status, and management implications (Doctoral dissertation), James Cook Univ.

20. Kulldorff M. 1997. A spatial scan statistic. Communications in Statistics: Theory and Methods 26: 1481-1496.

21. Kulldorff M. 2009. Informat Managem Serv, Inc.2009. SaT ScanTM v8.0: Software for the spatial and space-time scan statistics. http://www. satscan.org.

22. Lowe S, Browne M, Boudjelas S, Poorter M. 2000. 100 of the world's worst invasive alien species: a selection from the global invasive species database (Vol. 12). Invasive Species Specialist Group, Auckland.
23. MAyDS 2018.. Fortalecimiento de la gobernanza para la protección de la biodiversidad mediante la formulación e implementación de la estrategia nacional sobre especies exóticas invasoras. ENEEI, Minist. Amb. \& Desarr. Sustent., Argentina, GCP/ARG/023/GFF.

24. Navas JA. 1987. Los vertebrados exóticos introducidos en Argentina. Rev Museo Argent Cs Nat, Serie Zoología, XIV: 7-38

25. Oliver WL. 1993. Pigs, peccaries, and hippos: status survey and conservation action plan. Ed. IUCN, Vol. 19.

26. Oliver WL, Brisbin IL. 1993. Introduced and feral pigs: problems, policy and priorities. In: Status survey and conservation action plan: pigs, peccaries and hippos gland, William Oliver (ed.), Switzerland, p. 159-191.

27. Petrakovsky J, Carpinetti B, Antonuci A. 2016. Prevalencia serológica de leptospira sp en cerdos silvestres (Sus scrofa) en bahía Samborombón, Buenos Aires, Argentina, periodo 2013-2015. Salud y Tecnología Veterinaria 3: 23.

28. Sagua MI et al. 2018. Inferring the origin and genetic diversity of the introduced wild boar (Sus scrofa) populations in Argentina: an approach from mitochondrial markers. Mammal Res 63: 467-476.

29. Sanguinetti J, Pastore H. 2016. Abundancia poblacional y manejo del jabalí (Sus scrofa): una revisión global para abordar su gestión en la Argentina. Mastozoología neotropical 23: 305-323.

30. Schuyler PT, Garcelon DK, Escover S. 2002. Eradication of feral pigs (Sus scrofa) on Santa Catalina island, California, USA. Invasive Species Specialist Group, IUCN, Switzerland and Cambridge, UK, 274-286.

31. Tanger SM, Guidry KM, Nui H. 2015. Monetary estimates of feral hog damage to agricultural producers in Louisiana. Journal of the NACAA 8: 2.

32. Team QD. 2009. QGIS geographic information system. Open Source Geospatial Foundation, 117 p..

33. Tesón M, Regis A, Huici N, Novak F. 1997. Triquinelosis en jabalíes (Sus scrofa) en Lacar (Neuquén, Argentina). Rev Vet Arg 14: 187-190.

34. Villamil J, Krivokapich S, Ribicich M. 2013. Análisis epidemiológico de trichinellosis en humanos y jabalíes de La Pampa, Argentina. Alimentos y Zoonosis 8: 16.

35. Winter M et al. 2018. Trichinellosis surveillance in wildlife in northeastern argentine patagonia. Vet Parasit 11: 32-35. 